\title{
Experimental Results for Direction of Arrival Estimation with a Single Acoustic Vector Sensor in Shallow Water
}

\author{
Alper Bereketli, ${ }^{1}$ Mehmet B. Guldogan, ${ }^{2}$ Taner Kolcak, \\ Tamer Gudu, ${ }^{1}$ and Ahmet Levent Avsar ${ }^{1}$ \\ ${ }^{1}$ Underwater Acoustic Systems Engineering, METEKSAN Savunma San. A. S., Bilkent Cyberpark F-Building, \\ 06800 Ankara, Turkey \\ ${ }^{2}$ Department of Electrical and Electronics Engineering, Turgut Ozal University, 06010 Ankara, Turkey
}

Correspondence should be addressed to Alper Bereketli; abereketli@meteksan.com

Received 8 March 2015; Revised 29 April 2015; Accepted 29 April 2015

Academic Editor: Fanli Meng

Copyright (c) 2015 Alper Bereketli et al. This is an open access article distributed under the Creative Commons Attribution License, which permits unrestricted use, distribution, and reproduction in any medium, provided the original work is properly cited.

\begin{abstract}
We study the performances of several computationally efficient and simple techniques for estimating direction of arrival (DOA) of an underwater acoustic source using a single acoustic vector sensor (AVS) in shallow water. Underwater AVS is a compact device, which consists of one hydrophone and three accelerometers in a packaged form, measuring scalar pressure and threedimensional acceleration simultaneously at a single position. A very controlled experimental setup is prepared to test how well-known techniques, namely, arctan-based, intensity-based, time domain beamforming, and frequency domain beamforming methods, perform in estimating DOA of a source in different circumstances. Experimental results reveal that for almost all cases beamforming techniques perform best. Moreover, arctan-based method, which is the simplest of all, provides satisfactory results for practical purposes.
\end{abstract}

\section{Introduction}

This paper studies and compares the performarnces of several direction of arrival (DOA) estimation techniques using a single acoustic vector sensor (AVS) in shallow water. Formed by a hydrophone and three accelerometers, an AVS measures scalar acoustic pressure and vectorial acoustic particle acceleration at a location in space [1]. Pressure data is generally used as a phase reference to avoid sign ambiguity. Recent advances in AVS technology and DOA estimation techniques have enabled the usage of AVS devices in a wide range of real-time applications such as target localization [2], underwater defense [3], diver detection [4], underwater acoustic communication [5], and pipeline protection [6].

Beamforming results obtained with a single AVS and with a 16-element AVS array are compared in [7]. The focus of this work is to study the directional frequency analysis and recording (DIFAR) approach. An array of AVS devices is used to achieve DOA estimation and localization via acoustic pressure and acoustic particle velocity information [8]. Explicit expressions are derived for estimation errors for a multi-AVS model. Similarly, in [9], lower and upper performance bounds are studied for a complicated particle filtering approach to estimate two-dimensional DOA at an AVS. Analytical models are developed for source movement. Particle filtering techniques are also used for the removal of ambiguities related to the direction of an acoustic target on an AVS array [10]. In [11], the authors propose an algorithm which is based on angular diversity of incident acoustic waves on a three-dimensional AVS array. The algorithm exploits ESPRIT (Estimation of Signal Parameters via Rotational Invariance Techniques) and MUSIC (MUltiple SIgnal Classification) techniques for DOA estimation through velocity hydrophones and a pressure hydrophone. In [12], an adaptive recursive least-squares (RLS) method is used with an AVS, assuming that there is no multipath fading or acoustic interference. The solution requires a priori knowledge of incident signal power, source movement, and noise power. The DOA estimation algorithm in [13] makes use of subspace characteristics of the time variant covariance matrices of the uncorrelated quasi-stationary source signals. The model proposed in [14] aims at reducing memory requirements 
and computational cost of MUSIC-like algorithms used for AVS arrays. A data covariance model is proposed, and its outcomes for signal subspace estimation are investigated with numerical simulations. Another work [15] that focuses on avoiding the high computational cost of MUSIC algorithm obtains initial DOA estimations from the signal subspace and uses successive searches to achieve the DOA from an AVS array. The theoretical analysis in [16] shows that directional ambiguity problem occurring in DOA estimation results can be solved by using cross covariance matrices found in linear AVS array apertures. In order to detect the DOA of acoustic sources using an AVS array, a novel sensor array model is introduced in [17]. The model is based on higher-dimensional second-order statistics of received data at sensors.

Research efforts on DOA estimation using AVS are based on theoretical assumptions, without real measurement data. Studies based on experimental data are rarely found in AVS literature. In [1], pressure and acceleration outputs of an AVS are used to accomplish beamforming. Outputs of an AVS are used to estimate acoustic intensity vector, whose unit vector provides a DOA estimate for the acoustic source. The outputs are linearly combined to achieve spatial filtering, and the theory is validated through experimental results at Seneca Lake. Signal processing theory of AVS devices can also be used in public surveillance systems, as discussed in [18]. A method for automatic determination of position of speech signals and impulse sounds is presented. The events are detected in the presence of sound reflections employing acoustic vector sensors. The method for detection of acoustic events is found to be adequate for identifying sound sources inside auditory halls. Performed with a four-element vertical AVS array, the Makai experiment [19] provides valuable results in terms of DOA estimation, spatial filtering capabilities, and effects of parameters such as source range, depth, and seabed conditions. Conventional pressure-dependent estimation methods are extended to include particle velocity. Estimation performance is improved by a directivity factor that enhances side lobe reduction, demonstrating the advantage of using an AVS array over an array of hydrophones. The study in [20] aims at estimating the direction, range, and depth of an acoustic source with a single AVS, assuming that the environmental conditions of the channel are known. The proposed method is tested using simulated data and is further applied to experimental data from the Makai experiment, where $8-14 \mathrm{kHz}$ chirp signals were acquired by a vector sensor array [19]. The azimuth and elevation of echoes arriving on the AVS are estimated from particle velocity components using a least-squares algorithm.

Studies on AVS signal processing theory are mostly related to DOA estimation; however, they are not well suited for the unique features of real-time applications. The theoretical studies work on complex algorithms with demanding implementation, memory, and processing requirements. The results are based on assumptions or channel information based on hypothesis or theory rather than real measurement data. Moreover, some experimental studies using AVS provide very limited information regarding the performance comparison of different DOA estimation approaches. There are only a few studies on DOA estimation in an underwater environment $[1,7,19,20]$. Still, these studies present experimental data confined to the performance of a specific DOA estimation method applied on AVS arrays rather than a single AVS.

In order to meet the requirements of real-time systems which can be used in public or mission-critical applications, DOA estimation algorithms should be computationally efficient. Since digital signal processing hardware is practically limited in size, capacity, and cost, the performances of DOA algorithms must be investigated and compared using experimental data.

The objective of this study is to investigate and compare the performances of computationally efficient and relatively simple DOA estimation algorithms, namely, arctanbased method, intensity-based (IB) technique, time domain beamforming (TDBF), and frequency domain beamforming (FDBF), in a shallow water environment. These algorithms are selected according to our previous analysis [21] in which we compare the performances of DOA estimation techniques for a single underwater AVS under the effects of various error sources such as imperfect sensitivity patterns and ambient noise.

A very controlled experimental setup is prepared at the open water test-calibration facility of METEKSAN Savunma San. A. S. at Bilkent Pond. This paper presents the DOA estimation results of the mentioned techniques using a data set acquired during a series of experiments held in July 2014. HAARI VHS-90 [22] AVS is used in the experiments. The VHS-90 AVS provides a minimum sensitivity of $-200 \mathrm{~dB}$ re $\mu \mathrm{Pa}$ between 100 and $4000 \mathrm{~Hz}$. Its pressure sensitivity pattern is omnidirectional. The acceleration sensitivity on each axis shows a lateral rejection of at least $25 \mathrm{~dB}$ against other orthogonal axes. A GeoSpectrum M21 [23] projector is used as the acoustic source, transmitting a continuous sine wave at $4 \mathrm{kHz}$ from a distance of $5 \mathrm{~m}$. Both the source and the AVS are submerged to a depth of $5 \mathrm{~m}$, where the water depth is around $10 \mathrm{~m}$. It is important to note that multipath echoes affect DOA estimation performance adversely in such a shallow water environment [24]. Experimental results reveal that for almost all cases beamforming techniques perform best. Moreover, arctan-based method, which is the simplest of all, provides satisfactory results for practical purposes.

To sum up, the major contributions of this work can be itemized as follows.

(1) This paper studies the performances of several efficient DOA estimation techniques applied to real AVS measurements in shallow water. The experiments are challenging due to several reasons: (i) DOA estimation is performed with a single AVS instead of an array and (ii) the considered underwater environment brings acoustic propagation effects such as multipath disturbance, making it difficult to estimate DOA. To the best of our knowledge, this work is the first one in the literature in this respect.

(2) In addition to the theoretical discussion on DOA estimation algorithms, the paper presents real measurement data and practical performance results, which are very rare in AVS literature. 


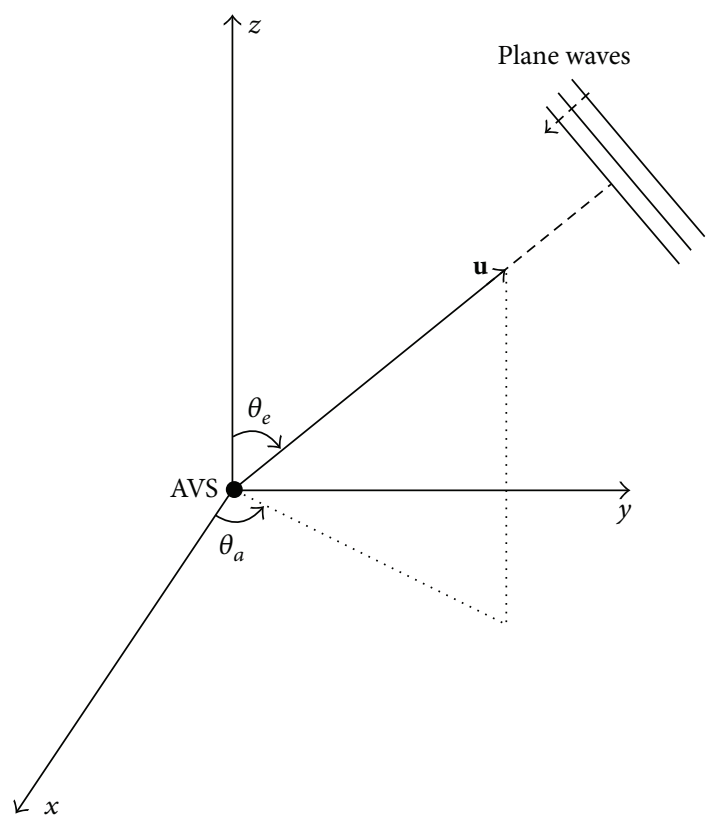

FIGURE 1: Projections of the unit vector at the AVS pointing towards the source.

(3) The comparison provides a valuable insight for the design and performance of practical AVS-based systems, where computationally simple and resourceefficient DOA estimation techniques are required.

The rest of the paper is organized as follows. Section 2 explains the theoretical framework for signal processing at an AVS. The DOA estimation algorithms used in our experiments are described in Section 3. The experimental setup, including the measurement environment and equipment, is presented in Section 4. Measured data and DOA estimation results are discussed. Section 5 concludes the paper.

\section{Sensor Model}

Throughout this study, we assume the far field condition holds, stating that the maximum wavelength is much smaller than the distance between the source and the AVS. In addition to the far field condition, the transmitter can be assumed to be a point source, since its dimensions are small compared to the acoustic wavelength. Thus, as shown in Figure 1, the wavefront incident on the AVS can be considered as planar. The AVS is located at the origin. At any time $t$, it measures the pressure, $p(t)$, and the orthogonal components of acoustic particle acceleration, $a_{x}(t), a_{y}(t)$, and $a_{z}(t)$.

The unit vector pointing towards the acoustic source is shown by

$$
\mathbf{u}=\left[\begin{array}{c}
\cos \theta_{a} \sin \theta_{e} \\
\sin \theta_{a} \sin \theta_{e} \\
\cos \theta_{e}
\end{array}\right]
$$

where $\theta_{a}$ and $\theta_{e}$ denote the azimuth and elevation angles of u, respectively. As discussed in [25], the acoustic pressure generated by a transmission at a frequency $f$ is given by

$$
p(\mathbf{r}, t)=P e^{j\left(\mathbf{k}^{T} \cdot \mathbf{r}-2 \pi f t\right)}
$$

at a point $\mathbf{r}=[x y z]$ in space. Here, the acoustic wave vector $-\mathbf{k}=\|\mathbf{k}\| \mathbf{u}$ is defined to point from the origin towards the acoustic source, and $\|\mathbf{k}\|=2 \pi f / c$ is the wave number. The amplitude of the acoustic wave pressure is represented by $P$. The pressure is related to the acoustic particle velocity vector through

$$
\mathbf{v}(\mathbf{r}, t)=-\frac{p(\mathbf{r}, t)}{\rho c} \mathbf{u},
$$

where $\rho$ is the density of the underwater medium and $c$ is the speed of sound in the medium [8]. The acceleration vector is obtained by [1]

$$
\mathbf{a}(\mathbf{r}, t)=\frac{\partial \mathbf{v}(\mathbf{r}, t)}{\partial t} .
$$

The projection of a on each axis is measured by the accelerometers placed orthogonally on the axes.

\section{DOA Estimation Algorithms}

The performances of computationally efficient and relatively simple DOA estimation algorithms, namely, arctanbased method, intensity-based (IB) technique, time domain beamforming (TDBF), and frequency domain beamforming (FDBF), are investigated. The discussion in [21] presents a detailed theoretical comparison of these algorithms for a single AVS with error sources such as imperfect sensitivity patterns and ambient noise. In this study, the algorithms are analyzed and compared using practical measurement data obtained in our shallow water experiments at Bilkent Pond.

3.1. Arctan-Based Technique. The simplest method to estimate the DOA of an acoustic signal is the arctan-based technique, which relies only on acceleration information and does not make use of the acoustic intensity on the AVS. Hence, the DOA for acoustic waves on $x y$ plane in Figure 1 can be estimated as

$$
\widehat{\theta}_{a}=\arctan \left(\frac{\sum_{n=0}^{N-1} a_{y}^{2}(\mathbf{r}, n T)}{\sum_{n=0}^{N-1} a_{x}^{2}(\mathbf{r}, n T)}\right)
$$

using the $x$ and $y$ components of the acceleration vector obtained through (1)-(4). Here, $N$ represents the number of samples used for estimation and $T$ shows the sampling period.

3.2. Intensity-Based (IB) Technique. The intensity-based technique uses the acoustic pressure incident on the AVS, as well as the acceleration vector. At time $t$, the acoustic intensity at a point $\mathbf{r}$ is expressed by the product of pressure and particle velocity [1]:

$$
\mathbf{I}(\mathbf{r}, t)=p(\mathbf{r}, t) \mathbf{v}(\mathbf{r}, t) .
$$




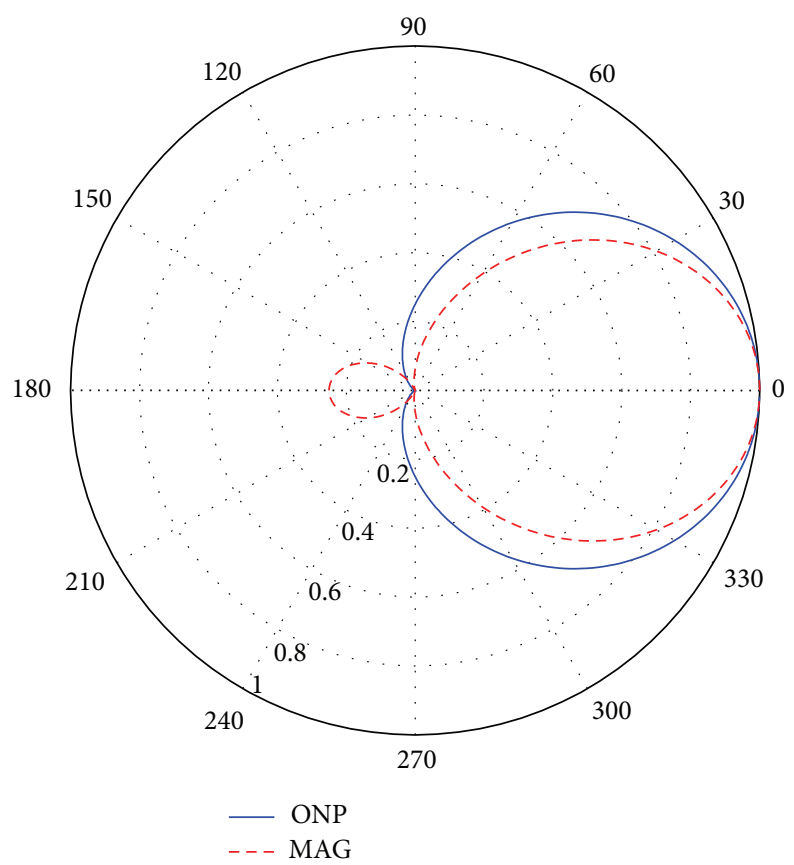

FIGURE 2: Beam shapes obtained with the coefficients in the beamforming technique.

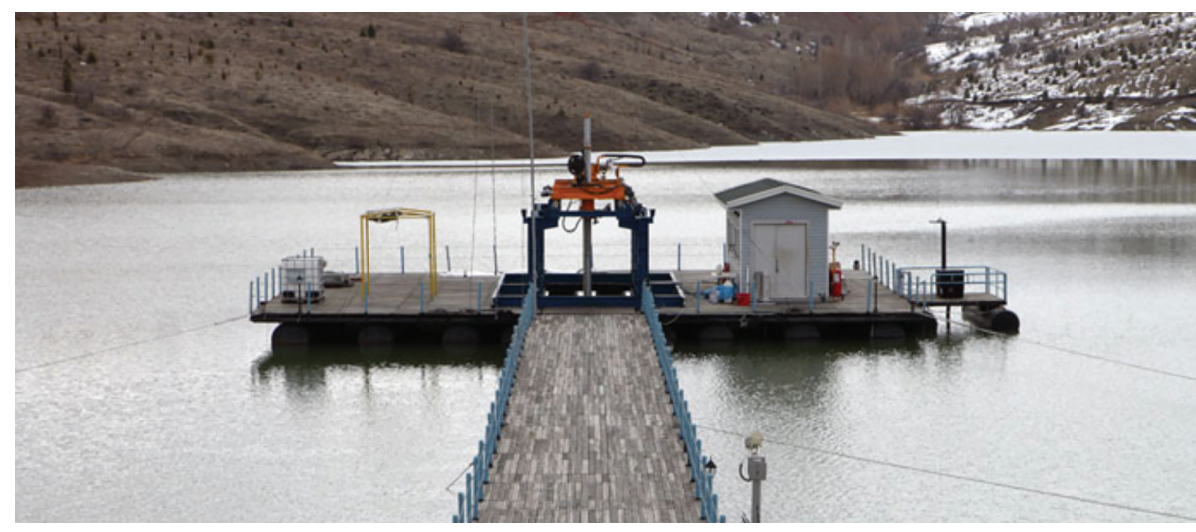

Figure 3: The open water test and calibration facility at Bilkent Pond.

Acceleration and velocity vectors are related through (4). In order to use acceleration and pressure data together for DOA estimation, we use

$$
\mathbf{J}=q(\mathbf{r}, t) \mathbf{a}(\mathbf{r}, t),
$$

where $q=\partial p / \partial t[1]$. To increase the signal-to-noise ratio (SNR), the IB technique uses a number of samples, constructing

$$
\mathbf{J}=\frac{1}{N} \sum_{n=0}^{N-1} q(\mathbf{r}, n T) \mathbf{a}(\mathbf{r}, n T)
$$

where $N$ is the number of samples taken with a sampling period $T$. Consequently, the DOA estimate for acoustic waves along the $x y$ plane can now be obtained by

$$
\widehat{\theta}_{a}=\arctan \left(\frac{J_{y}}{J_{x}}\right) \text {. }
$$

3.3. Beamforming Techniques. Beamforming techniques rely on pressure data as well as accelerometer measurements. Using $q(\mathbf{r}, t)$ and $\mathbf{a}(\mathbf{r}, t)$ given in (7), these measurements are multiplied by different weights to shape the resulting beams. Assuming that digital data samples are obtained with the abovementioned sampling period $T$ for a location $\mathbf{r}$, the discrete-time output signal constructed with time domain beamforming (TDBF) is

$$
\begin{aligned}
s[n]= & w_{0} q\left[n-\tau_{0}\right]+w_{1} a_{x}\left[n-\tau_{1}\right]+w_{2} a_{y}\left[n-\tau_{2}\right] \\
& +w_{3} a_{z}\left[n-\tau_{3}\right],
\end{aligned}
$$

where $w_{0}, w_{1}, w_{2}$, and $w_{3}$ are the angular weights of the corresponding intensities and $\tau_{0}, \tau_{1}, \tau_{2}$, and $\tau_{3}$ are the temporal shifts depending on beam direction [1].

Frequency domain beamforming (FDBF) is accomplished by simply taking the Discrete Fourier Transform 

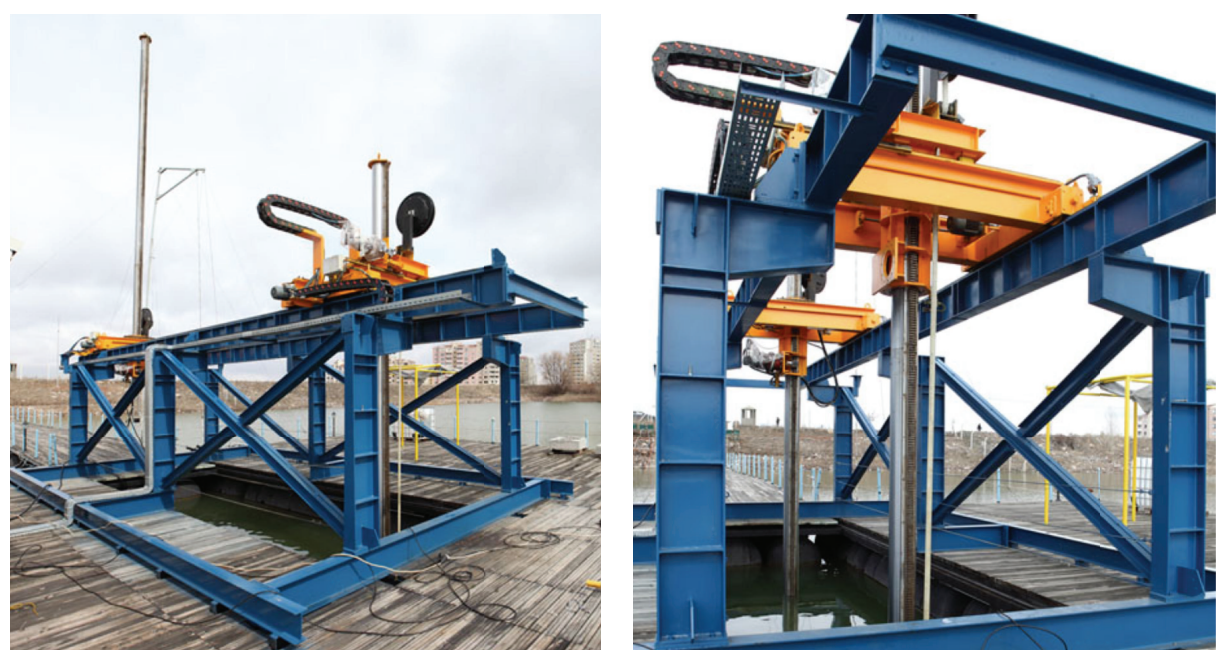

Figure 4: Measurement setup from two different angles. Relative positions of the source and the sensor are adjusted by controlling the mechanical structure (orange part).

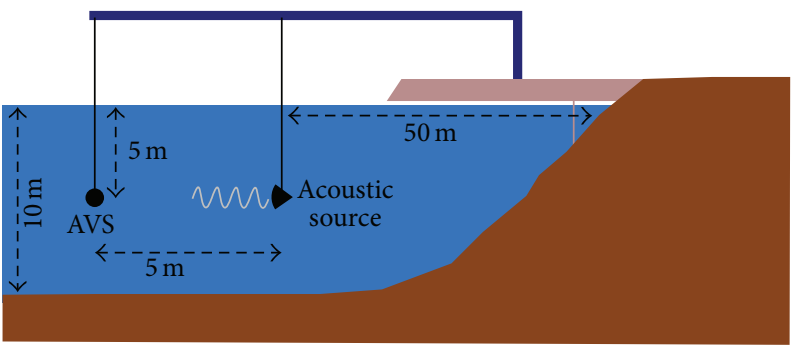

Figure 5: Illustration of the experimental setup at Bilkent Pond.

(DFT) of (10) over $N$ samples at each angle, obtaining the following frequency domain signal expression:

$$
\begin{aligned}
S[k]= & \omega_{0} Q[k] e^{-j 2 \pi k \tau_{0} / N}+\omega_{1} A_{x}[k] e^{-j 2 \pi k \tau_{1} / N} \\
& +\omega_{2} A_{y}[k] e^{-j 2 \pi k \tau_{2} / N}+\omega_{3} A_{z}[k] e^{-j 2 \pi k \tau_{3} / N},
\end{aligned}
$$

where $S[k]$ is given by

$$
S[k]=\sum_{n=0}^{N-1} s[n] e^{-j 2 \pi k n / N} .
$$

Summing $Q$ and $\mathbf{A}$ with different weights result in different beam patterns.

In this work, we use a beam shape that gives the optimum null placements (ONP), as described in [1]. To achieve ONP in the constructed beam, the weights in (10) can be calculated as

$$
\begin{aligned}
& w_{0}=\frac{a_{0}}{\rho c} \\
& w_{1}=a_{1} \cos \left(\theta_{s}\right) \sin \left(\phi_{s}\right) \\
& w_{2}=a_{2} \sin \left(\theta_{s}\right) \sin \left(\phi_{s}\right) \\
& w_{3}=a_{3} \cos \left(\phi_{s}\right),
\end{aligned}
$$

where $\theta_{s}$ and $\phi_{s}$ are steering angles for azimuth and elevation, respectively, in accordance with Figure 1.

The ONP beam shape does not yield the maximum array gain (MAG). However, the MAG beam is more focused in the direction of reception but tends to yield a lobe in the opposite direction [1]. In low-SNR cases, this reverse lobe may lead to sign ambiguity in azimuth estimation. To get rid of the sign ambiguity, the ONP shape, which has a wider beam width without the reverse lobe, is used in our study. The undesired lobe is avoided with zero phase shift, that is, $\tau_{0}=\tau_{1}=\tau_{2}=\tau_{3}=0$, and by taking $a_{0}=a_{1}=a_{2}=a_{3}=1[1]$. These beam shapes are shown in Figure 2, where it is assumed that $\phi_{s}=90^{\circ}, \theta_{a}=0^{\circ}$, and $\theta_{e}=90^{\circ}$; that is, there is a plane wave traveling along the $x$-axis towards the AVS at the origin.

\section{Experimental Setup and Results}

A very controlled experimental setup is prepared at the open water test and calibration facility of METEKSAN Savunma Sanayii A. S. at Bilkent Pond, as shown in Figure 3. The pond is approximately $300 \mathrm{~m}$ long by $100 \mathrm{~m}$ wide, and the measurement structure, which is presented in Figure 4, is placed at a distance of $50 \mathrm{~m}$ to the shore. Water depth below the structure is around $10 \mathrm{~m}$. The bottom type is mud in general. Regarding the adverse effects of surface multipath echoes in shallow water [24], the measurement 

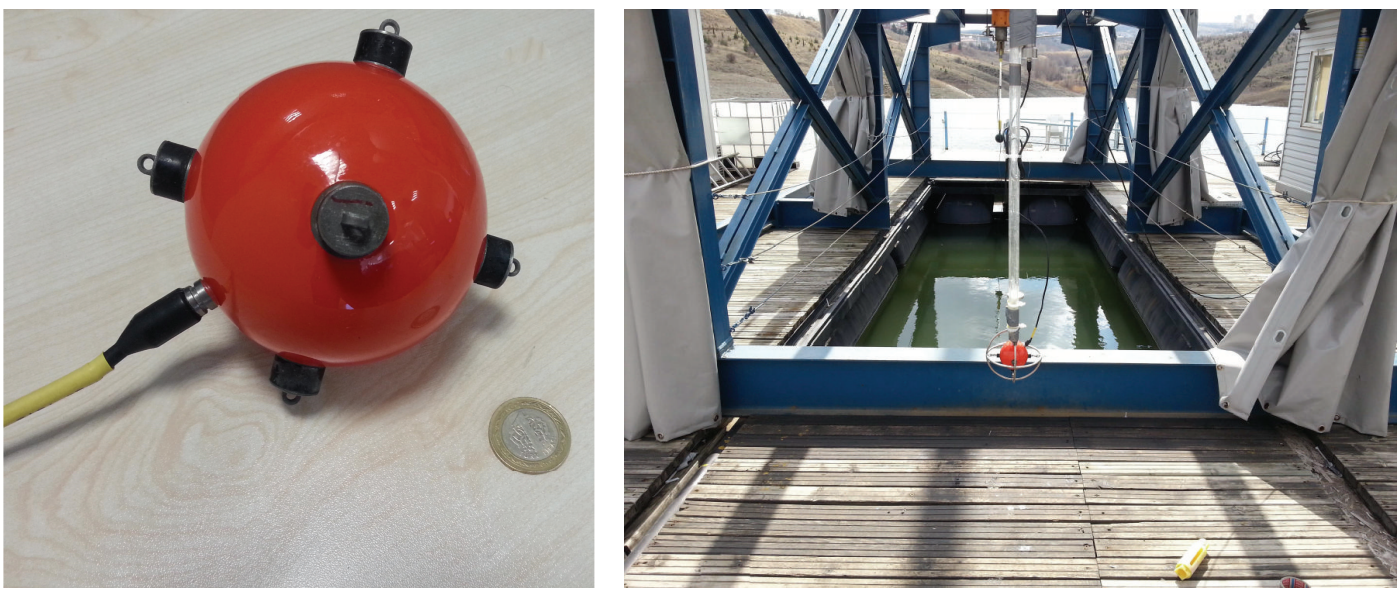

FIGURE 6: The HAARI VHS-90 AVS used in the experiments, mounted at the measurement structure.
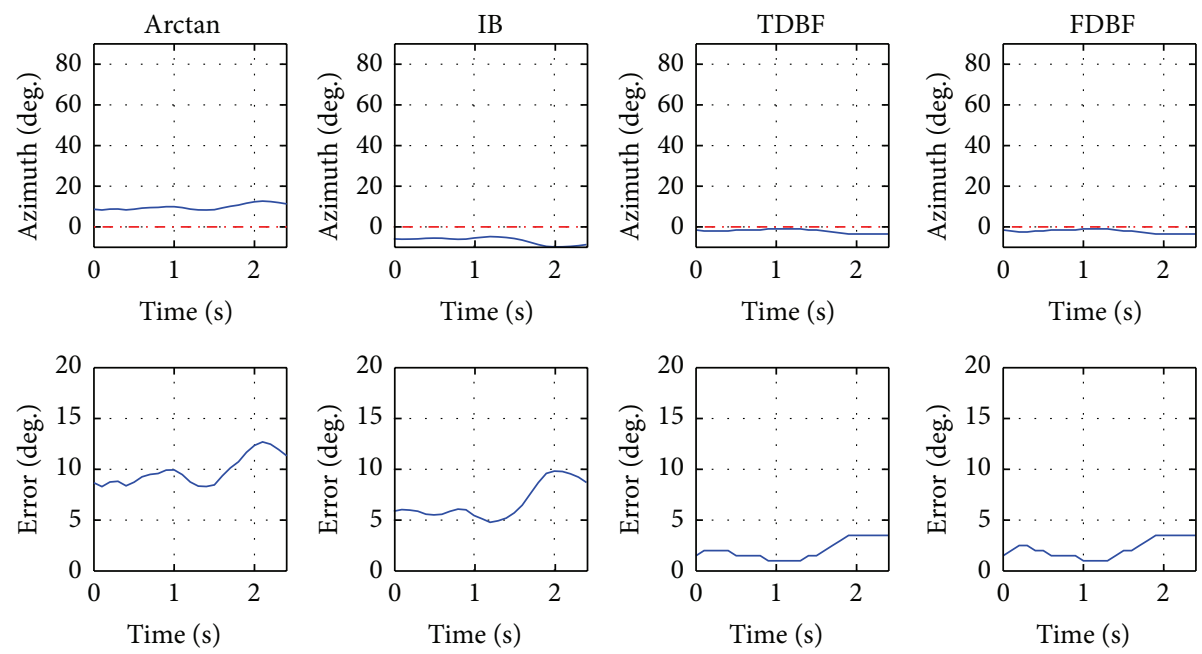

(a)

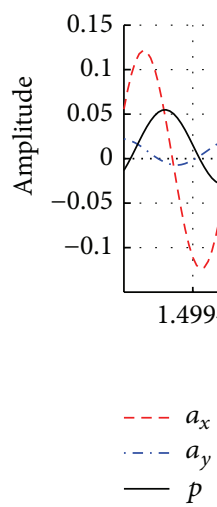

(b)

Figure 7: (a) Estimation results and errors and (b) measurement data zoomed into a $1 \mathrm{~ms}$ interval for $\theta_{a}=0^{\circ}$. Ground truth is shown by the dashed red line in (a).

environment is challenging in evaluating the performances of DOA estimation algorithms. This paper presents the DOA estimation results of the algorithms using a data set acquired during a series of experiments that took place in July 2014.
As illustrated in Figure 5, both the acoustic source and the AVS are suspended off the measurement structure at $5 \mathrm{~m}$ depth. Since they are at the same depth, we are dealing with the azimuthal DOA estimation of $\theta_{a}$. The source is a 

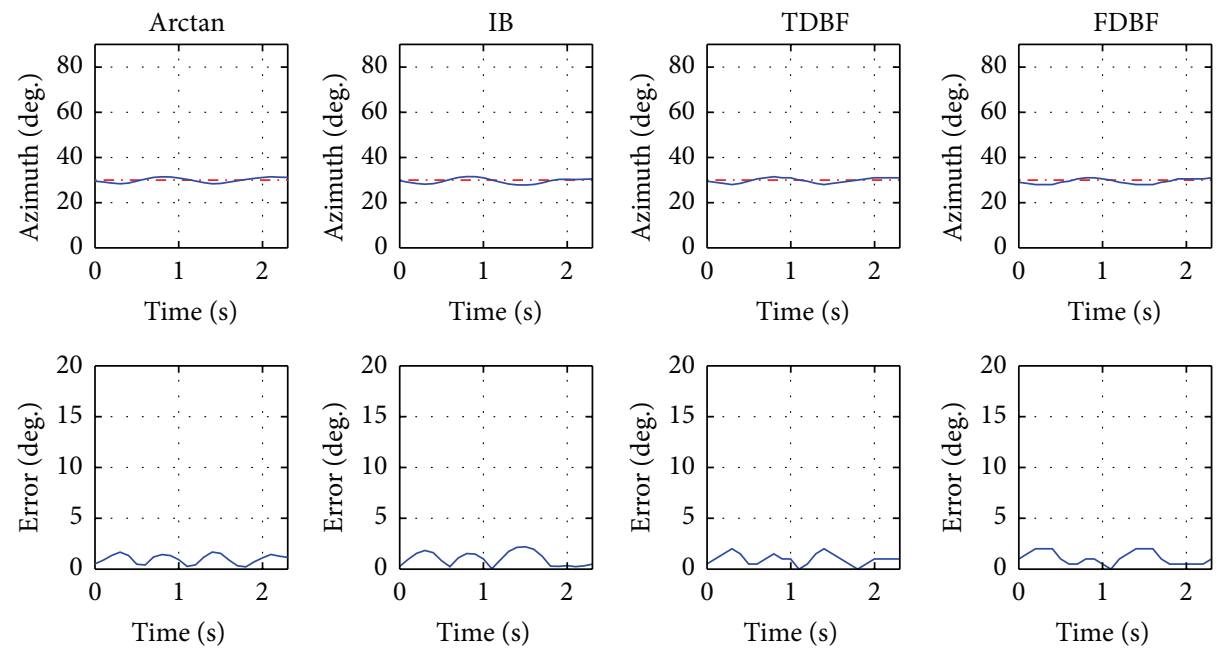

(a)

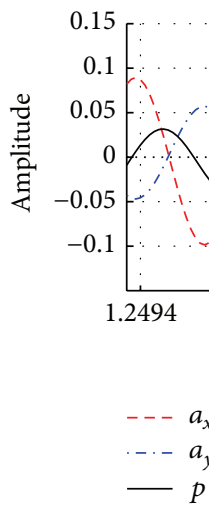



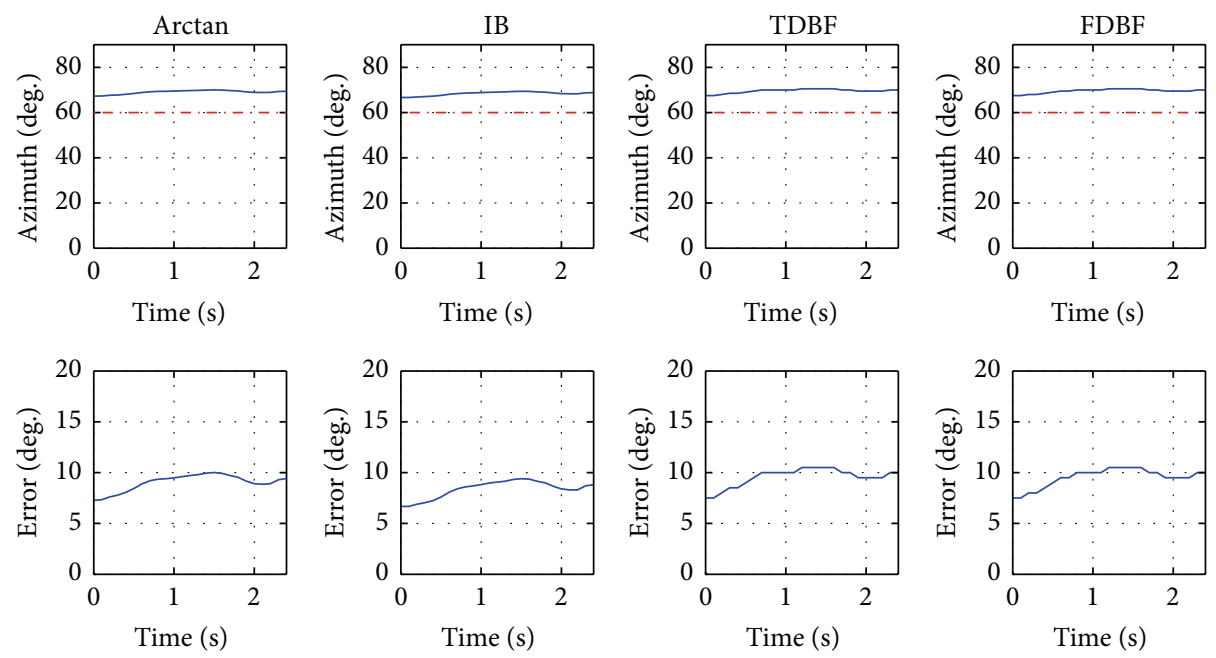

(a)

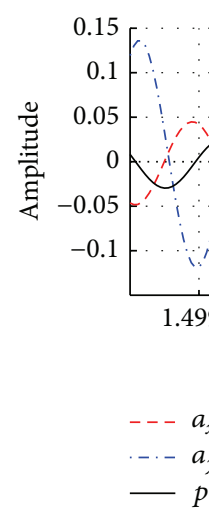

(b)

FIGURE 9: (a) Estimation results and errors and (b) measurement data zoomed into a $1 \mathrm{~ms}$ interval for $\theta_{a}=60^{\circ}$. Ground truth is shown by the dashed red line in (a).

The objective of the measurements is to compare the performances of computationally efficient, easy to implement, and relatively simple two-dimensional DOA estimation algorithms, namely, arctan-based, IB, TDBF, and FDBF techniques.

Figures 7-10 show the measurement results and errors as well as measurement data for several directions between $\theta_{a}=0^{\circ}$ and $\theta_{a}=90^{\circ}$. In almost all measurements, TDBF and FDBF techniques perform better than the other algorithms. Due to a technical problem in the data acquisition system, we failed to retrieve pressure data successfully for $\theta_{a}=$ $90^{\circ}$ (Figure 10(b)). We intentionally included this case to show how the performances of the mentioned techniques are affected. In such problematic cases, the IB algorithm, which depends on pressure as well as acceleration data, performs poorly when compared to the other DOA estimation methods. Although the arctan-based method is the simplest of the four investigated algorithms, it generally offers satisfactory estimation performance for practical applications. Even when pressure data is not obtained successfully, beamforming techniques yield the best estimation results due to their frequency selectivity and angular directivity.

\section{Conclusions}

This paper presents and compares performances of arctanbased, intensity-based, time domain beamforming, and frequency domain beamforming methods in estimating the DOA of an underwater acoustic source using a single AVS in shallow water. For this purpose, an experimental setup is configured and real measurement results are collected at the open water test and calibration facility of METEKSAN Savunma Sanayii A. S. at Bilkent Pond. In shallow water, it is known that the propagation is dominated by multipath echoes which degrade DOA estimation performance. The paper presents real measurement data and practical performance results, which are infrequent in the literature. Experimental results show that beamforming techniques perform better than arctan-based and intensity-based methods. It is observed that the simplest approach, arctan-based technique, provides satisfactory performance. The comparison provides a valuable insight for the design and performance of practical AVSbased systems, where computationally simple and resourceefficient DOA estimation techniques are required. 

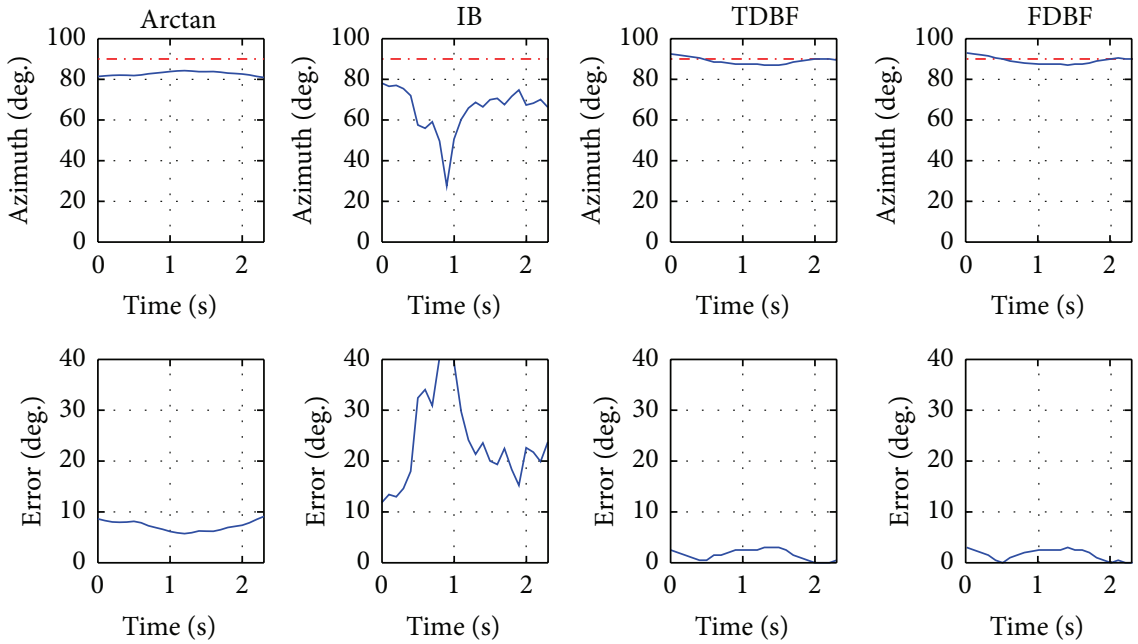

(a)

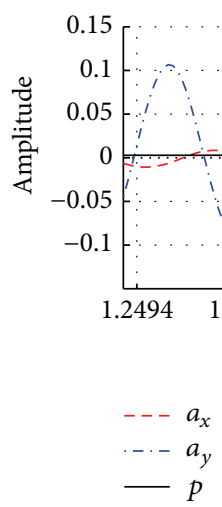

(b)

FIGURE 10: (a) Estimation results and errors and (b) measurement data zoomed into a $1 \mathrm{~ms}$ interval for $\theta_{a}=90^{\circ}$. Ground truth is shown by the dashed red line in (a).

\section{Conflict of Interests}

The authors declare that there is no conflict of interests regarding the publication of this paper.

\section{References}

[1] M. T. Silvia and R. T. Richards, "A theoretical and experimental investigation of low-frequency acoustic vector sensors," in Proceedings of the MTS/IEEE (OCEANS '02), vol. 3, pp. 18861897, Biloxi, Miss, USA, October 2002.

[2] M. Hawkes and A. Nehorai, "Wideband source localization using a distributed acoustic vector-sensor array," IEEE Transactions on Signal Processing, vol. 51, no. 6, pp. 1479-1491, 2003.

[3] J. A. Clark and G. Tarasek, "Localization of radiating sources along the hull of a submarine using a vector sensor array," in Proceedings of the MTS/IEEE (OCEANS '06), pp. 1-3, Boston, Mass, USA, September 2006.

[4] R. N. Carpenter, B. A. Cray, and E. R. Levine, "Broadband ocean acoustic (BOA) laboratory in narragansett bay: preliminary insitu harbor security measurements," in Photonics for Port and Harbor Security II, Proceedings of SPIE, Orlando, Fla, USA, April 2006.
[5] A. Abdi, H. Guo, and P. Sutthiwan, "A new vector sensor receiver for underwater acoustic communication," in Proceedings of the MTS/IEEE OCEANS, pp. 1-10, IEEE, Vancouver, Canada, September 2007.

[6] X. Song, Z. Jian, G. Zhang, M. Liu, N. Guo, and W. Zhang, "New research on MEMS acoustic vector sensors used in pipeline ground markers," Sensors, vol. 15, no. 1, pp. 274-284, 2015.

[7] G. D’Spain, W. S. Hogkiss, G. L. Edmonds, J. C. Nickles, F. Fisher, and R. A. Harriss, "Initial analysis of the data from the vertical DIFAR array," in Proceedings of the MTS/IEEE OCEANS, pp. 346-351, IEEE, Newport, RI, USA, October 1992.

[8] A. Nehorai and E. Paldi, "Acoustic vector-sensor array processing," IEEE Transactions on Signal Processing, vol. 42, no. 9, pp. 2481-2491, 1994.

[9] X. Zhong, A. B. Premkumar, and A. S. Madhukumar, "Particle filtering and posterior Cramér-Rao bound for 2-D direction of arrival tracking using an acoustic vector sensor," IEEE Sensors Journal, vol. 12, no. 2, pp. 363-377, 2012.

[10] P. Braca, P. Willett, K. LePage, S. Marano, and V. Matta, "Bayesian tracking in underwater wireless sensor networks with port-starboard ambiguity," IEEE Transactions on Signal Processing, vol. 62, no. 7, pp. 1864-1878, 2014. 
[11] K. T. Wong and M. D. Zoltowski, "Self-initiating MUSIC-based direction finding in underwater acoustic particle velocity-field beamspace," IEEE Journal of Oceanic Engineering, vol. 25, no. 2, pp. 262-273, 2000.

[12] M. K. Awad and K. T. Wong, "Recursive least-squares source tracking using one acoustic vector sensor," IEEE Transactions on Aerospace and Electronic Systems, vol. 48, no. 4, pp. 3073-3083, 2012.

[13] S. Zhao, T. Saluev, and D. L. Jones, "Underdetermined direction of arrival estimation using acoustic vector sensor," Signal Processing, vol. 100, pp. 160-168, 2014.

[14] S. Miron, N. Le Bihan, and J. I. Mars, "Quaternion-MUSIC for vector-sensor array processing," IEEE Transactions on Signal Processing, vol. 54, no. 4, pp. 1218-1229, 2006.

[15] Z. Xiaofei, Z. Ming, C. Han, and L. Jianfeng, "Two-dimensional DOA estimation for acoustic vector-sensor array using a successive MUSIC," Multidimensional Systems and Signal Processing, vol. 25, no. 3, pp. 583-600, 2014.

[16] Y. Wu, G. Li, Z. Hu, and Y. Hu, "Unambiguous directions of arrival estimation of coherent sources using acoustic vector sensor linear arrays," IET Radar, Sonar \& Navigation, vol. 9, no. 3, pp. 318-323, 2015.

[17] K. Han and A. Nehorai, "Nested vector-sensor array processing via tensor modeling," IEEE Transactions on Signal Processing, vol. 62, no. 10, pp. 2542-2553, 2014.

[18] J. Kotus, K. Lopatka, and A. Czyzewski, "Detection and localization of selected acoustic events in acoustic field for smart surveillance applications," Multimedia Tools and Applications, vol. 68, no. 1, pp. 5-21, 2014.

[19] M. Porter, B. Abraham, M. Badiey et al., "The Makai experiment: high frequency acoustics," in Proceedings of the 8th European Conference on Underwater Acoustics (ECUA '06), pp. 12-15, Carvoeiro, Portugal, June 2006.

[20] P. Felisberto, O. Rodriguez, P. Santos, E. Ey, and S. M. Jesus, "Experimental results of underwater cooperative source localization using a single acoustic vector sensor," Sensors, vol. 13, no. 7, pp. 8856-8878, 2013.

[21] A. Gunes, M. B. Guldogan, and A. Bereketli, "A comparative study on the performances of the DF techniques using a single acoustic vector sensor," in Proceedings of the MTS/IEEE (OCEANS '14), pp. 1-7, St. John's, Canada, September 2014.

[22] Hangzhou Applied Acoustic Research Institute (HAARI), March 2015, http://www.hariutc.com/en/.

[23] Geospectrum Technologies, 2015, http://geospectrum.ca/.

[24] R. J. Urick, Principles of Underwater Sound, McGraw-Hill, New York, NY, USA, 1983.

[25] B. A. Cray and A. H. Nuttal, "A comparison of vector-sensing and scalar-sensing linear arrays," Tech. Rep. NUWC-NPT, 1997. 

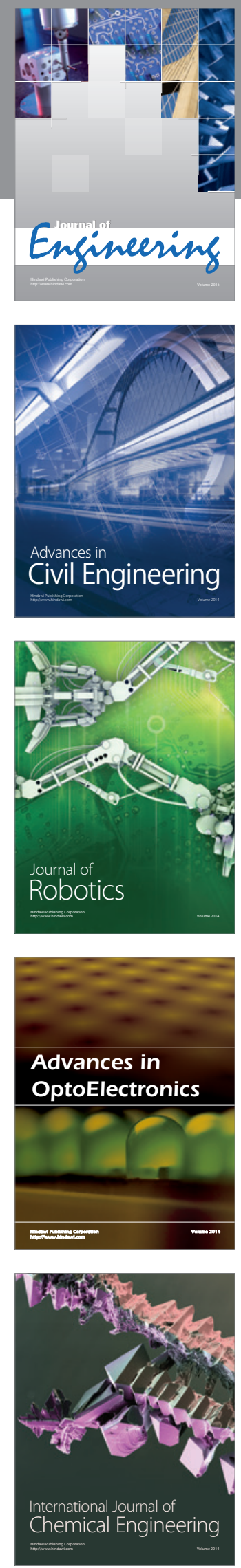

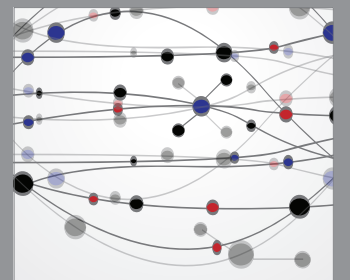

The Scientific World Journal
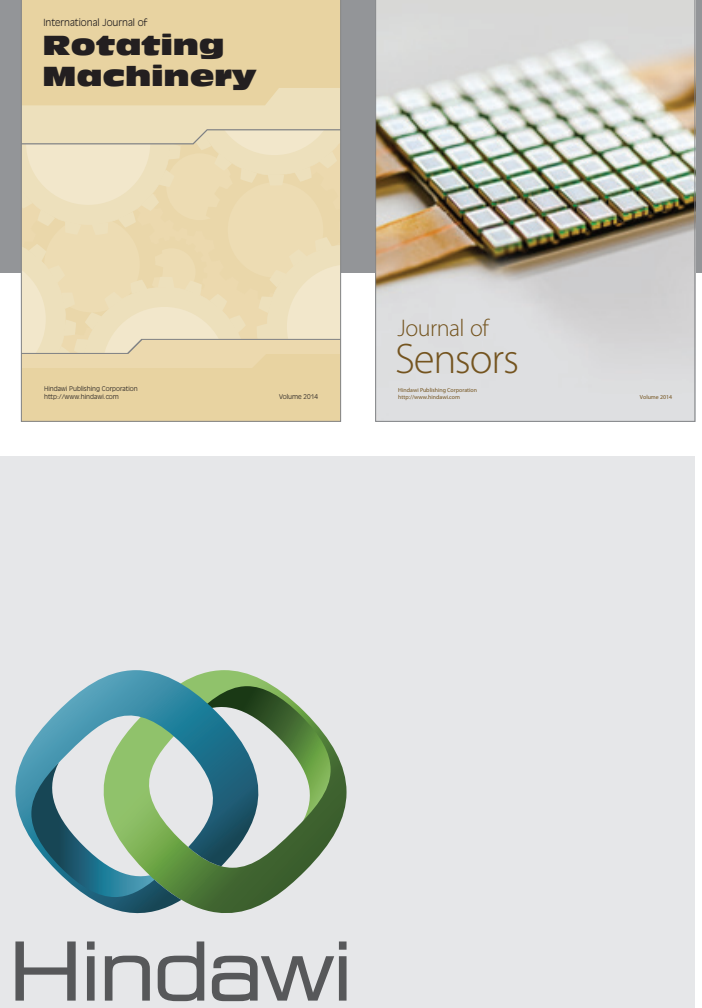

Submit your manuscripts at http://www.hindawi.com
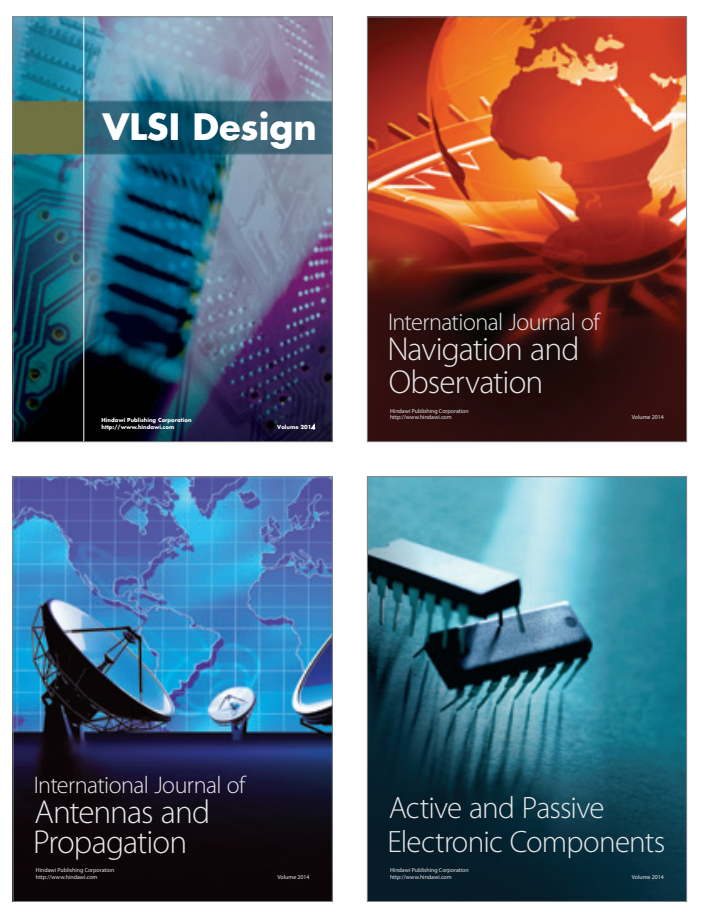
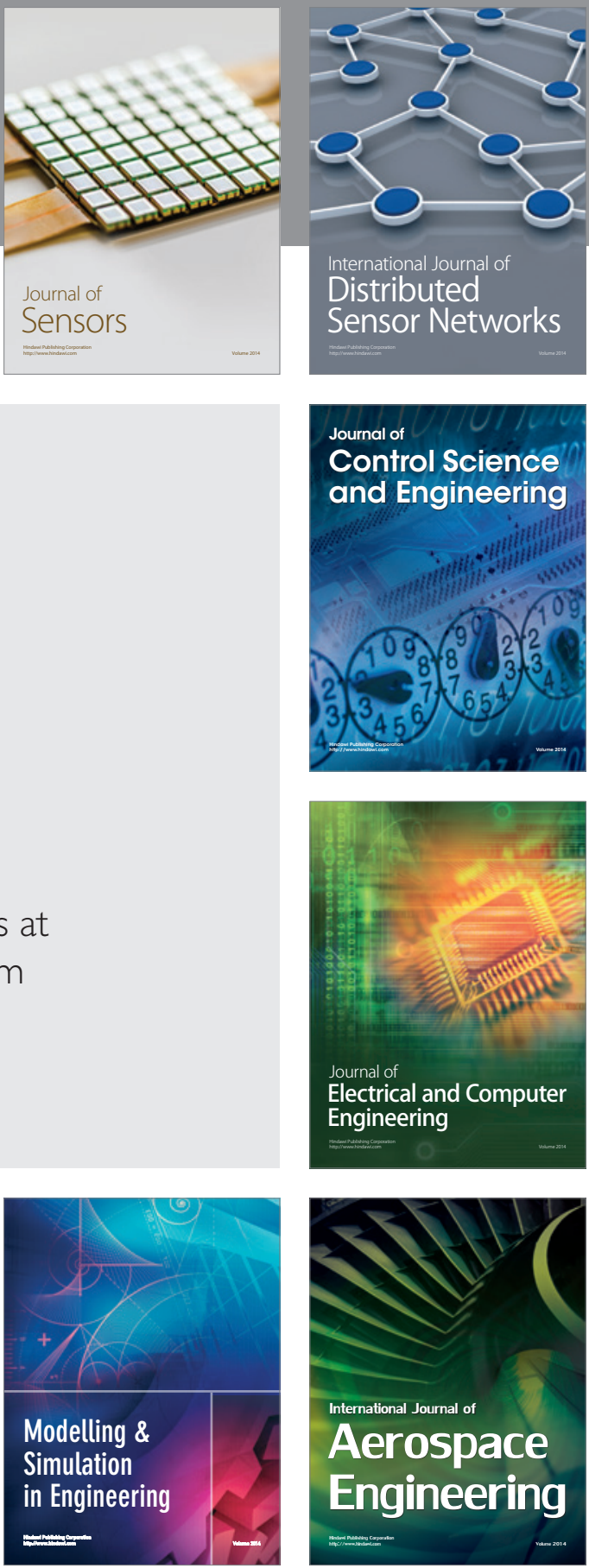

Journal of

Control Science

and Engineering
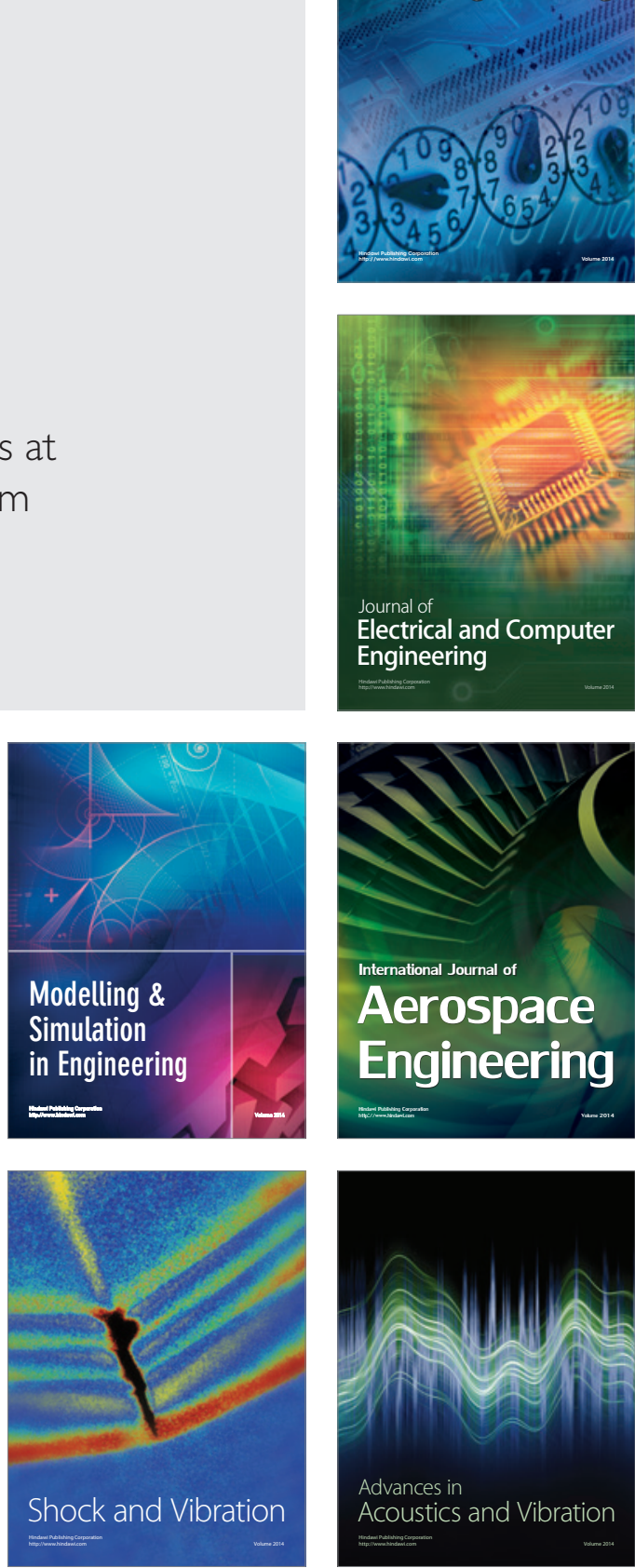